\title{
Minority Stress for Care Experienced Young Queer People: A Case Study
}

\author{
Lynne McPherson $^{1}$ (1) $\cdot$ Kathomi Gatwiri $^{1} \cdot$ Nadine Cameron $^{2} \cdot$ Janise Mitchell $^{3}$
}

Accepted: 29 October 2021 / Published online: 16 November 2021

(c) The Author(s), under exclusive licence to Springer Nature Switzerland AG 2021

\begin{abstract}
Young people who are placed in out-of-home care are amongst the most vulnerable in our community. Removed from or rejected by their families, they must learn to live with carers who may be strangers. They may have experienced the trauma of abuse or neglect. Post care, they may experience further social isolation and marginalisation due to limited social capital. These challenges are compounded for queer young people placed in out-of-home care. This study adopted a case study approach to explore the lived experience of two young adults growing up queer in and out of out-of-home care in Australia. Our investigation was framed by two complementary theoretical frameworks. The first, a model of minority stress, is informed by queer perspectives and enabled an exploration into the adverse impact of enduring stigma and prejudice associated with homophobia and transphobia on young people's capacity to thrive. The model of social capital was then employed to inform an analysis of relationships between queer young people in out-of-home care and trustworthy adults. The findings suggest that queer young people growing up in out-of-home care experience minoritised stress, with lasting negative implications. The presence however, of trustworthy adults who challenge dominant heteronormative assumptions and work to support queer young people can be experienced as stabilising and restorative.
\end{abstract}

Keywords Queer · Young people · Out-of-home care · Foster care · LGBTIQA+

\section{Highlights}

- Queer young people in out-of-home care can experience stress associated with sexual identity minoritization, compounded by their 'in care' status

- Queer young people in out-of-home care may lack supportive social connections

- Adults playing a key role in the queer young person's life can facilitate the development of their social capital

$\triangle$ Lynne McPherson
Lynne.mcpherson@scu.edu.au
$\triangle$ Kathomi Gatwiri
Kathomi.gatwiri@scu.edu.au
$\triangle$ Nadine Cameron
Nadine.cameron@scu.edu.au
Janise Mitchell
jmitchell@ childhood.org.au
$1 \quad$ Faculty of Health, Socal Work, Southern Cross University,
Lismore, Australia
2 Faculty of Health, Southern Cross University, Lismore,
Australia
Australian Childhood Foundation, Richmond, Australia

\section{Introduction}

Evidence suggests that young people in out-of-home care often come from troubled family backgrounds, where a safe and secure base is absent (Beek \& Schofield, 2004). They manifest complex needs, which are underpinned by histories of the trauma of abuse, abandonment, neglect and deprivation (Gatwiri et al., 2018; McPherson et al., 2018). Because experiences of child abuse and neglect are intersectional and informed by variables such as age, race, gender, disability and sexual orientation, queer youth in care are likely to have additional trauma history or challenges due to their minority sexual orientation and gender identities (Schofield et al., 2019). LGBTIQA + is an acronym that represents lesbian, gay, bisexual, transgender, intersex, queer/questioning, asexual and a number of other terms, including non-binary and pansexual, to describe people's experiences of gender, sexuality and sex characteristics (Australian Institute of Family Studies, 2019). An umbrella term, 'queer' describes a range of sexual orientations and gender identities and, as such, will be adopted for use throughout this paper. 
The specific needs of queer youth in out-of-home care systems appear to have been under-researched. Agencies supporting young people in out of home care have, in some instances, produced helpful guidance for staff working with LGBTIQA + youth (CREATE, 2021), however a dearth of scholarly knowledge may contribute to less-than-safe practices which "have been complicit in causing additional trauma and poor outcomes" for this cohort of young people (Cook \& Cohen, 2018, p.1). Unlike other identifying characteristics such as race, age and gender, sexual orientation still remains on the margins of demographic data in child welfare systems (Woronoff et al., 2006). In the United States, queer youth are overrepresented in the child welfare system and report significant health disparities due to "experiences of discrimination, marginalization, and an overall lack of acceptance" (McCormick et al., 2017, p. 27). The actual prevalence of queer youth in out-of-home care in Australia and elsewhere is unclear, due in part to the stigma associated with 'coming out' (McCormick et al., 2017). Young people in care are likely to be highly scrutinised by staff members due to the common misconception that LGBTQ youth are more likely to sexually abuse other young people in care (Brandon-Friedman et al., 2020).

As Meyer (2003) argues, the prejudice and social stress associated with living with a minoritised sexual identity can be traumatic for queer young people in care. Queer youth are more likely to be rejected by family members (meaning a lesser chance of being reconciled/reunited with family), less likely to receive medical and mental health services that are affirming, more predisposed to depression, likely to have a higher number of suicide attempts and more likely to use addictive substances; they also register higher rates of psychopathology (Cochran et al., 2002; Ryan et al., 2009).

It is now well recognised that the transition from care for young people universally may be extraordinarily challenging, given their lived experience of abuse and neglect in the care of their family and for many, whilst in the care of the State (Mendes \& Snow, 2016). This transition for queer youth, however, may bring even more challenges. Cochran et al. (2002) report that they have higher rates of risk factors on a range of health and behavioural measures and poorer socio-economic outcomes compared to heterosexual young people. Queer youth are over-represented in homeless shelters, and remain there for longer periods, with those exiting the care system experiencing additional stress and obstacles (Coolhart \& Brown, 2017). These negative outcomes demonstrate that more attention with regard to the sexual orientation and gender identity of queer young people in out-of-home care would be critical in creating a safe environment. Mallon and Woronoff (2006, p. 118) suggest that "the welfare of LGBT children, youth, and families cannot be adequately enhanced as long as the larger society, heterocentrically oriented and heterosexually controlled, ignores their existence". Additionally, Woronoff et al., (2006, p. 6) argue that:
LGBTQ youth need to feel that they are not condemned and stigmatized for something they cannot change and that is an important part of them-their sexual orientation or gender identity. They need to be cared for in an environment that shields them from, not exposes them to, bias and prejudice. They need to be free from anti-LGBTQ slurs and harassments. They need to feel loved, not hated, for who they are. They need to know that the child welfare system and adults who care for them are on their side.

Unfortunately, the invisibility of research and practice guidelines for working with queer youth in out-of-home care can contribute to the erasure of their experiences by practitioners and other peers (McCormick et al., 2017). Put simply, a significant gap in the research surrounding queer youth may be attributed to the lack of knowledge and understanding among practitioners about the lived experiences of queer youth in out-of-home care. In Australia, this research is in its infancy. The current study begins to address that gap.

\section{Theoretical Frameworks}

Two theories were integrated to frame this study. The first, a 'minority stress' model which is informed by queer perspectives and sociological and psychological theories, attempts to conceptualise the "adverse effect of social conditions, such as prejudice and stigma, on the lives of individuals and groups" from minority groups (Meyer, 2003, p. 675). The stress of enduring prejudice and the discrimination of sexual identity minoritization in the form of homophobia or transphobia can strain an individual's capacity to cope and thrive. This model enables us to explore whether and to what extent queer young people in out-of-home care experience an exacerbation of minoritised stress due to their sexual orientation and gender identities. The second, an adaption of social capital theory, has a focus on how and if affirming relationships with adults taking on the role of "empowerment agents" can facilitate the development of a healthy and positive self-regard (Barker \& Thompson, 2015). The two theories (discussed below), in tandem, enabled an in-depth exploration of young peoples' lived experience in out-ofhome care.

\section{Minority Stress for Care Experienced Young Queer People}

In considering queer perspectives, traditional categorisations of gender which view heterosexuality as the standard norm of sexual expression and identity are deemed as oppressive and limiting. The universalisation of heteronormativity erases queer experiences in society, including in 
out-of-home care systems. Queer perspectives probe heteronormativity as a cultural system and a location of privilege that structures social life around it. This is, for example, through the structuring of families, relationships, sexuality and identity, and is seen as a social construction that produces heteronormative binaries. Chevrette (2013, p. 172) argues that the "signifier, 'queer' disrupted the homosexual/ heterosexual binary, countered categorical models of sexual identity, and examined the cultural, historical, and political discourses around which sexuality is constructed". As such, in recognising the expressions of queer identities and practices and the embedded hetero practices, structures and systems that shape our world, we utilise minority stress theory to argue that the continued stress of minoritization, under the weight of heteronormativity, impacts on the psychological, mental and social wellbeing of queer young people in out-of-home care.

This theoretical framework highlights how chronic, psychosocial stressors related to discrimination, stigma and victimisation inform the negative health outcomes of queer young people (Kelleher, 2009). The social external structures embedded in the heteronormative scripts of our society shape the social and self-identities of queer people. Meyer (2003) suggests that there are three processes of minority stress that inform the experiences of queer individuals. These are "(a) external, objective stressful events and conditions (chronic and acute), (b) expectations of such events and the vigilance this expectation requires, and (c) the internalization of negative societal attitudes" (p. 676). An identity that is constantly under attack and threatened by prejudice and discrimination is strained and exhausted by the continued exertion of energy that is required to maintain one's sense of self-identity and self-concept. Within this, an intersectional conceptualisation of minority stress is also necessary. Black, Indigenous and queer young people of colour face double marginalisation due to their race and sexual orientation (Bowleg et al., 2003). Minority stress explores how identities are shaped socially through the process of self-categorisation. This means that when a particular group is viewed and therefore categorised as different from the acceptable norm, it triggers processes of exclusion and marginalisation.

For young people in out-of-home care, minority stress may be experienced through rejection, abuse, prejudice and discrimination from peers, caregivers and other adults. Although overt discrimination is frowned upon, covert heteronormative practices that are still embedded in child welfare institutions can exclude the unique needs of queer youth. Wilson et al., (2014, p. 11) argue that in the child welfare system, practices of prejudice against queer youth are commonly expressed through them being deemed as difficult to foster, and/or blamed for expressing their sexuality, which can lead to isolation to avoid them "preying on other youth" or for "their own safety". Because of these continued practices, the child welfare system is not often viewed as a safe and supportive place for queer youth (Woronoff et al., 2006). In fact, the homophobia, transphobia and heterosexism embedded in society and institutions of child welfare problematize queer youth, while at the same time erasing their unique experiences and making them feel invisible (Woronoff et al., 2006, p. 6). This reveals that, unwittingly or otherwise, the dominant child welfare policies and practices can serve to exclude queer youth in care. Woronoff et al., (2006, p. 9) argue that in order to improve the experiences of queer youth, "combating homophobia and the heterosexist assumptions that pervade the child welfare system should be the number-one priority".

\section{Social Capital Theory}

Trustworthy relationships take on central significance for the developing young person in out-of-home care. The theory of social capital asserts that relationships that are "resources and key forms of social support embedded in one's network or associations, and accessible through direct or indirect ties with institutional agents" (Stanton-Salazar, 2011 p. x). The concept of an 'institutional agent' (Stanton-Salazar, 2011) facilitating the development of 'linking social capital' (Barker \& Thompson, 2015) is central to this framework and is defined as an individual who occupies one or more hierarchical positions of authority. Such an individual, situated in a young person's social network (for example, caregiver, coach or teacher), manifests their potential role as an institutional agent when, on behalf of the young person, they act to directly transmit (form supportive relationship), or negotiate the transmission of, highly valued resources (e.g. access to education, contact with family and friends, housing and support) (Barker \& Thompson, 2015). Whilst the significance of relationships is theorised, relationships alone may not constitute 'capital' (Barker \& Thompson, 2015). Three critical components must exist for a relationship to be realised as social capital. Firstly, there needs to be contact with the person or people; secondly, this connection must enable access to valued resources; and thirdly, the connection takes place in the context of a trusting, reciprocal relationship (Barker \& Thompson, 2015).

For young people in out-of-home care, we theorise that such 'agents' could be carers, teachers or other adults who both understand and enact direct and negotiated transmission of valued relationships and resources and practise in ways that promote social justice and challenge oppressive or unjust regulations or requirements. Given the highly problematic lived experience faced by young queer people in out-of-home care, drawing on theories of minority stress and social capital uniquely positions this research to investigate identified problems and potential solutions. 


\section{Methodology}

The aim of this research, based on the identified literature and theoretical framework, was to explore the experience of young queer people living in out-of-home care.

\section{Study Design}

A case study approach was selected to generate an in-depth understanding of the experiences of queer young people in out-of-home care, following the methodological path identified by Yin (2009). Case studies do not endeavour to identify 'typical' cases, nor make comparisons across cases, but do seek to develop an understanding of the phenomenon being studied (Yin, 2009). Considering the dearth of research involving this cohort in an Australian context, this approach, founded within an overall qualitative paradigm, emphasises the role of the researched in analysis and interpretation (Yin, 2009). A key focus of the approach is the way in which people make sense of their lived experience and their world whilst understanding that subjective human understandings of events and experiences may change over time and may be sensitive to difference in social contexts.

\section{Sampling, Recruitment and Data Collection}

A recruitment advertisement was placed on mainstream social media, circulated by LGBTIQA youth support organizations and posted on queer youth friendly social media sites. To be eligible to participate, they had to be young adults (18-29 years) who identified as queer and had experience of being in out of home care. After 8 months of a targeted recruitment strategy which included consultation with LGBTIQA + advocacy groups, organizations and community leaders, only four people responded with only two of those being able to meet the criteria for inclusion. Ethical approval to conduct the research was granted by the Southern Cross University Human Ethics Committee: project number 2019/169.

A schedule for in-depth interviews was developed by the research team based on the identified research question and in close consultation with an identified co-researcher who identified as young and queer with lived experience of outof-home care. Emerging from this consultation the following broad questions were agreed for the interview schedule.

(i) Reflecting on what brought you into out of home care... what do you believe you needed most at that time? To what extent were those needs met or not met? (ii) Reflecting on your relationships whilst in care, how were they experienced by you and with whom?

(iii) What helped or enabled you to develop your sense of sexual and gender identity?

Consistent with a semi-structured interview design, each question served as a discussion starter for the respondent to elaborate upon and raise and relevant issues as they chose to. Researchers then sought to clarify and extend responses by inviting respondents to elaborate. These in-depth interviews were conducted digitally using zoom technology as a response to the safety concerns surrounding the COVID19 global pandemic restrictions and face-to-face contact. Informed written consent from participants was obtained via email prior to the interviews. Following each of the interviews, which lasted between 2 and $2.5 \mathrm{~h}$, participants were provided with a draft transcript for comment or correction.

\section{Analysis}

Data analysis was undertaken using both deductive and inductive strategies. Both inductive and deductive, or "theoretical" thematic analysis processes were used to organize and present the data (Braun \& Clarke, 2006, p.84). A six-step process was followed which included: a) becoming familiar with the data, b) individual research team members generating initial codes based on the aim of the study, c) grouping codes into emerging themes as a team of researchers, d) discussing and reviewing the themes, e) integrating the named themes with theoretical frameworks and f) constructing the report. This method enhanced the theoretical validity as the research team initially worked to code independently before discussing and agreeing on the emerging themes. The theoretical thematic analysis was undertaken as a parallel process, enabling researchers to code for and focus on the data in the context of minority stress theory (Meyer, 2003) and linking social capital theory (Barker \& Thompson, 2015). At the completion of analysis undertaken by the team of three researchers, themes were identified and reported on.

\section{Results: A Snapshot of the Participants}

What follows is a de-identified snapshot, of each of the young-adult research participants. Pronouns used are consistent with those preferred by each. We have used pseudonyms throughout this manuscript to protect the respondent's privacy. The first participant, Liam, (he/him) identified as 'proudly queer', was aged between 20 and 30 years, and was strongly involved in promoting social justice on LGBTIQA + issues via community and social 
media platforms. Liam, along with his three younger siblings, was removed from the care of his parents in suburban Australia when he was around six years of age. The initial removal followed a series of reports to the statutory child protection service concerning family violence and concerns about parental mental health. Liam reported that his father was "extremely violent" and that his mother struggled with severe depression and suicidality. Having been removed from home, he then spent a number of years living in five different foster care placements, alternating with trial periods back at home. He recalled a particularly distressing incident: a phone call to let him know that a parent had died as a result of suicide. Despite the placement and relational instability that Liam experienced, he noted that he was almost always able to remain at the same school and, on all but one occasion, was placed together with his siblings. For the most part, Liam recalled a sense that he was constantly just 'trying to survive' emotionally whilst in various placements, never quite fitting in or included in family life. One point of difference in his care trajectory was a short-term placement with two women who were said to be 'friends' living together. Liam experienced care from this couple that was warm, nurturing and nonjudgemental. Following an extended period in his fourth or fifth foster care placement, Liam briefly returned home to live with his father as a teenager. He subsequently moved to live independent of his family and the care system and has now been in a stable living arrangement for almost a decade.

The second young adult, Tim, (he/him) identified as an Aboriginal transgender male. He was aged between 20 and 30 years and his contact with out-of-home care was intermittent from the age of nine years where he was in informal placements with either a grandparent or a family friend for extended periods. The concerns leading to these placement arrangements were the experience of physical abuse, where on one occasion Tim was hospitalised with a severe injury. The experiences of placement, both with his grandparent as a kinship carer and a family friend as a 'kith' carer, were experienced by Tim as continuous and stable relationships with extended family, rather than as "removal" and placement within the care system. He recalled fondly as he talked about his grandmother who taught him about his culture and accompanied him by train and bus to school each day. Despite this stability, Tim identified a number of health challenges and complex personal needs that are ongoing due to his childhood adversity. At the time of the interview, he was living in his "former foster sibling's apartment", offered as a safe space throughout the COVID-19 global pandemic. Tim was pursuing a higher degree in research at an Australian University.

\section{Thematic Discussion}

Overall, findings suggested that early adversity, marginalisation and the violence of homophobia whilst in care and/or within the wider community were directly experienced by Liam and Tim. Both readily recalled and recounted aspects of their lived experience of out-of-home care with a level of distress. Themes emerging from in-depth interviews suggest the pervasive presence of marginalisation and an exacerbation of minoritised stress, considering intersections of developing queer identities with race, early adversity and being different because they did not live at "home". Three core themes were identified and are discussed below.

\section{Theme 1: Minoritised Stress Associated With Homophobia and Heteronormativity for Young Queer People}

Both Liam and Tim spoke of the challenge to consider their sexual and gender orientations whilst living in care, given the considerable emotional demands of surviving emotionally on a 'day to day' basis. Whilst both said that they knew that they were queer whilst in care, it was not 'safe' to come out for some years. For Liam, it was not until he began to live independently, aged 18 years that he publicly identified as queer, initially to his housemates. For Tim, however, it was a two-stage process where he first identified as queer to his (supportive) kinship carer, however did not come out as Trans until he was at university, and also living independently. The implications of this theme are discussed on three levels: homophobia, invisibility/ lack of appropriate support, and stigma and discrimination. At the first level of consideration, we argue that the homophobia that queer youth face takes a psychological toll. This affects young people's sense of self, forcing them, in part, to deny and repress the expression of their sexual identities (Woronoff et al., 2006). In outof-home care, homophobia is demonstrated through overt actions of violence that may be enacted verbally, physically or psychologically. Youth in care can be the "easy" target of violence by their peers (Cameron et al., 2019), yet are consequently labelled as "provocative or aggressive" if they fight back or defend themselves (Mallon, 1992, p. 551). The fear of being outed or experiencing homophobic bullying contributes to negative mental health outcomes for queer youth in out-of-home care. Tim reflected on the normalisation of heterosexuality, as well as homophobic violence he experienced growing up, as follows:

There was so much [homophobic] bullying, and there's a lot of acceptance (of that) in high schools. In the university I was at, I was able to finally find those commu- 
nities, which was really helpful. I came out as bisexual when I was 21 but I knew I was Trans when I was 18, but that was when I had first heard the term.

Other forms of homophobia were more covert and can be enacted through silence and the lack of representation of non-hetero bodies in popular culture, books, media and communities. Liam reflected that:

I think, growing up ... it was a very homophobic environment. I didn't have any role models. I didn't have any family who were queer. My mother grew up in a very conservative Irish Catholic family. Went to the best all girls' school in the state. She was quite proper, and so was my Nan. I never felt comfortable, even with my parents.

Bringing lived experience as an Aboriginal young person, Tim discussed heteronormativity as a form of a violent colonial ideology which sought to binarize people. In his description, Indigenous worldviews on gender pre-colonization were fluid and not confined through the performativity of gendered expectations. Tim stated:

Being First Nation, we experience gender in a very different way. The gender binaries arrived with colonization. It wasn't in our culture before white people came in and applied it to us. We have different cultural roles in our society which aren't based on gender, it's based on whether you have a more nurturing spirit or a more warrior, fighting spirit. It had nothing to do with gender, but then white men came in and said, nurturing ah, must be women, warrior, must be men.

Tim's experience and reflection compel us to further theorize the impact of colonization on Australian First Nations people. It has been suggested that Indigenous Australians have been constructed as being "straight" only since European settlement, with accounts of Aboriginal sexual and gender diversity "mostly absent in the recordings and interpretations of histories" (Bayliss, 2014, p. 1). Added to this complexity is the dominant Eurocentric expectation that Indigeneity implies a lack of sexual and gender diversity. For a queer Aboriginal adolescent already struggling with their developing identity the weight of colonization bore heavily in this context and adds to the impact of minority stress.

Whilst there may be an increased acceptance of LGBTQ + people in society, stigma-based victimisation and marginalisation remain a serious problem. The stigma emanating from homophobia or transphobia is often expressed not just in personal interactions but is also embedded in the larger social and structural practices of out-of-home care. Experiences of queer youth in out-ofhome care reflect the culture of heteronormativity which reinforce feelings of "being different". Consistent stigma resulting from homophobia or transphobia can result in queer youth viewing themselves as unlovable, bad or different. Tim argues that "the most challenging thing was handling other people's curiosity and other people's discrimination and stigma". Experiences of stigma, which include bullying, homophobic micro aggressions, harassment, disapproval, victimization or violence, are associated with poor mental health outcomes.

Tim reflects that "I knew I was queer when I was younger but didn't come out" because "the worst thing you could be called was gay or a lesbian. There was that whole thing of, 'eww, that's disgusting that person must be a lesbian because that's so gross". Gower et al. (p. 2) argue that "the more frequently enacted stigma is experienced and/or in multiple contexts, the greater the likelihood of proximal stressors (e.g. social withdrawal, identity concealment, and internalised homophobia) and the more likely one is to experience minority stress".

One impact of the perceived stigma associated with identifying as queer for Liam was a delay in publicly identifying as queer until he had left the care system, his family home and his teenage years behind:

Oh no, I didn't come out until I was 20. Dad was a very religious person growing up, he and his first wife were very religious, and they played in the band at church. Even though he was an absolute arsehole, isn't that the way, the ones that preach the loudest are usually the pricks?

The stigma and homophobia are compounded by the fact that there are few services that offer safe and appropriate services to queer youth in out-of-home care. Our findings support international evidence that queer youth face substantial additional challenges compared to their heterosexual counterparts in the welfare system. Generally, there is an 'invisibility' and lack of appropriate support which impact on the development of a positive sexual identity. Mallon et al. (2002) found that, on average, queer youth in out-of-home care settings receive fewer services than their heterosexual counterparts. Liam reflected on the lack of appropriate resources that might have enabled him to positively reflect on his identity;

There were never any children's books, or any discussions about attraction or playing with boys or playing with girls, it was still really hush, hush ... The teachers can only teach, and then the parents need to reinforce it at home. And if it's not reinforced in school, it's the parents reinforcing it, usually from their own experiences of oppression, marginalization, stereotyping.

Liam recalled that, in his experience, there were so many ways in which he felt invisible or 'different', relating those 
experiences to the lack of visibility of queer people in foster care and the wider community:

Talking about feeling different, we didn't even have that (information and resources) around mental health. We barely do now, ... but back then we didn't even have books about depression or trauma and what it all means. Or to be in out of home care. I think lots of things could have been done, not just at a foster care level, but also at a family first and child protection level and within the school level.

The lack of available, visible and accessible support was a concern for Tim, who found it harder to reconcile his gender identity in a timely way. Due to the pervasiveness of homophobic or transphobic environments, queer youth must first assess the safety of 'coming out' or disclosing their sexual identity, which adds to the daily stressors of minoritization (The Center for the Study of Social Policy, 2016). Many fear that the disclosure will be accompanied by rejection or abuse. McCormick et al. (2017) argued that queer youth report being treated differently as soon as foster carers, peers or staff learn of their sexual orientation. In our case study, Tim reflected that he was "too scared to come out because [he] couldn't find any support for Trans people". He added that having to hide his sexual identity for fear of their safety "was really hard because I was hiding this part of myself for years". In their study, Ragg et al. (2006, p. 259 ) found that many queer youth organize their lives in a way that ensures the least likelihood of rejection. This means adopting strategies like withholding their identity, dating outside of their gender preference, self-isolation and internalizing homophobia as a way into the heteronormative "ideal".

Studies report that due to their inexperience or discomfort of working with queer youth, practitioners can consistently question their sexual identity in an effort to "convert" or "return them to the normal" heterosexual identity (Jenkins \& Johnston, 2004; Ragg et al., 2006, p. 247). The lack of services for Trans people contributes to their invisibility within the welfare system. Although Tim argues that things are "getting better now", he adds that "trans people are [still] almost invisible". In the event that these professional support services are available, the majority are deemed "incompetent and unresponsive" to the needs of queer youth (Ragg et al., 2006, p. 246). Tim found that:

The structured supports that were in place that I could find, they were transphobic, there just wasn't anything specifically for Trans people. I was [later] involved with 1911 (pseudonym- a queer support and advocacy organization) but even their resources were quite limited.

Oakley and Bletsas (2018) argue that the lack of appropriate provision of services for this population is due to the lack of recognition of their identities as queer. Australian researchers reported that within the broader service provision landscape, young queer people are not sufficiently catered for (McNair et al., 2017). Queer young people in outof-home care often describe a system in which homophobia, transphobia and general ignorance about the needs of this population are pervasive.

\section{Theme 2: Relationships Facilitating the Development of Social Capital}

It is essential that queer youth feel cared for by adults who are well informed and who make active efforts to unlearn any homophobic biases they may hold and engage with the young people from a place of unconditional positive regard and acceptance. Affirming relationships validate the queer experience by acknowledging the struggles of living through and with a minoritised sexual identity. Ragg et al. (2006, p. 258) argue that "when the [LGBTQ] youth describes concerns or feelings, having them affirmed as real and important helps them integrate their internal reality into their identity". Tim suggested that the affirming and loving relationships that he had with his carer (Susie) and grandmother made a positive difference to his overall experience of care. $\mathrm{He}$ reflected:

I think I needed different things from both Susie and my grandma. And I think I got those things from both of them which is really nice. From grandma, when I was a kid I acted like a boy and I thought I was a boy. And growing up I didn't know what transgender was and what it meant. And I didn't know anything about the sexual and gender diverse community. Susie knew quite a bit about the LGBTI community, and she really welcomed that conversation which was really lovely ... If I'd been with anyone else in out of home care, that conversation could have gone very differently.

This narrative indicates the active and available presence of a trusting relationship, acting clearly as an 'agent' for Tim in relation to his developing identity and social capital (Stanton-Salazar, 2011). Susie challenged the status quo, which for Tim was a homophobic school culture, whilst offering an emotionally safe space for Tim, when he was ready, to have a conversation more directly about his own developing identity.

In Liam's experience, affirmation from adults came from a brief experience of care with a lesbian couple. Liam notes that the couple introduced themselves as 'friends', which he later attributed to a wider issue of homophobia in the 1990s in suburban Australia. Unlike other placements where he recalled feeling different, marginalised and bullied, this placement is recalled with fondness: 
I don't know, they just made me feel very welcome. They used to drive us around a lot. They had a minivan. They drove us to all the activities we had to do ... Maybe it was just having two women around as well. I always felt more comfortable around women. Perhaps because my dad was very abusive during that time. I don't have any traumatic memories from my time with Anne and Sophie, whereas the other caregivers I had negative experiences ... They seemed very together, and I liked that.

Whilst the relationship between this couple and Liam was not to be an ongoing one, it offered a level of warmth and nurturing that he did not experience in other, hetero-sexual placement relationships. It is theorised that this experience was a useful resource for Liam as he began to explore his own sexual identity. Liam also experienced some adults in his school communities as affirming and safe. For example, some teachers took care of him over the weekends in the event of emergency situations until another foster care placement could be established. These relationships offered the stability that his foster care arrangements (with multiple placements) did not. Relationships with teachers also affirmed and encouraged his academic and creative talents. Liam thrived and extended himself academically, describing himself as "always a clever kid";

My teachers were very supportive of me ... And even some of the teachers took care of us as foster carers during some traumatic times as well. Some of the teachers would look after us over a weekend period where we needed to be immediately removed and there were no foster placements. We had a real community around us. The teachers did become like our parents in a way. I think that's important to note as well. Primary school was our only real stability.

The power of the school as a resourceful community is evidenced by these stories. Teachers who were willing to identify and build on young people's identified strengths, such as engagement in drama and the arts for Liam, offered an important source of reassurance and validation. The experience of literally being cared for (in emergency placement situations) in addition to being cared about offered Liam a sense that he was worthy and connected to a community. On the other hand, Tim articulated an absence of queer or Indigenous community support throughout school and the overwhelming sense of relief he experienced when he eventually connected with a local queer support service in the community that he described as a "lifesaver". However, he still struggled to connect with a queer group that would also affirm his Indigeneity:

Because I came out when I was already at university, I was able to find the queer community on campus, so the Queer Collective and the queer party scene and all that which was really, really useful. [However] ... There was no one who could really connect with me. There were a few people who worked there who were People of Colour, and they could kind of get it from their perspective as being a Person of Colour, but there was no one with a First Nations background. There was no one there to talk to about what it was like to be a Trans person and how to navigate cultural activities and how to come to terms with being Trans in your culture in comparison to being Trans and being white.

Both Liam and Tim identified connection to adults (teachers or carers) and communities that were affirming and trustworthy, offering a stable presence and an alternative perspective to the homophobic views and attitudes that were prevalent within the community. These relationships were transformative for both. Trusted adults and safe communities challenging homophobic views and attitudes, directly or indirectly, offered a powerful and empowering resource for Liam and Tim. Relationships with these 'agents' seem to have assisted both, to some extent, to work toward not only claiming their queer identity with pride, but proactively advocating for global change and, for the most part, enjoying stable and connected lives.

\section{Theme 3: Overcoming Adversity Through Advocacy}

Both Tim and Liam spoke with pride about their accomplishments as young adults who have become active in advocacy roles in a number of ways. In describing their involvement in the community, each identified their current connection to social justice and the desire to create a world that was a safer place for queer young people. Tim reflected that he was part of the committee that designed and created the Trans Hub Community. He is also highly engaged in social media as a way to connect with other socially isolated queer youth. He adds:

I do Instagram takeovers and I do interviews [on those platforms]. I am always advocating. Because if something like that was out when I was dealing with my identity it could have saved me years of pain and confusion.

Liam also spoke with great personal insight about both his activism and his career choice to enter the helping professions:

It [working in the helping professions] came very naturally to me. It felt comfortable and safe. I liked the idea of being a counsellor and using my experiences of empathy and vulnerability in my work. And then 
I realised it was really advocacy, what I was doing in my work. Then I became very agitated about the world, and I began to exercise a lot of my experiences through my practice and the years of internalising the effects of my story.

Recognising the complexity of identity and circumstance, Liam also noted that:

I am privileged. I'm able to use my maleness, my whiteness, my privilege, my education, but also my vulnerableness and those marginalised identities to drive and fuel some of the work I do which can support those spaces where people feel they might not feel welcome.

Despite the adversity faced by Liam and Tim prior to and throughout their care experiences, both have made considerable gains post-care in their respective educational and/ or employment achievements, voluntary and/or advocacy work, and housing and relationship stability. They both report strong and supportive relationships with some family members and excellent educational outcomes.

\section{Implications for Researchers and Practitioners}

The damaging impact of homophobic attitudes, values and beliefs for people who identify as queer is gaining some recognition globally (Brandon-Friedman et al., 2020). In addition, some international research highlights the difficulties experienced by queer young people in out-of-home care who may face multiple forms of marginalisation as a result of intersecting vulnerabilities and difference. An important consideration for practitioners emerging from this study is the power of trustworthy relationships with adults who hold the power to challenge dominant hetero-normative narratives, as well as social capital in the form of affirming queer-safe communities that can be easily accessed when needed. This case study found that such relationships and communities were pivotal in enabling queer young people to feel that nothing was 'wrong' with them. Developing an identity that is neither stigmatised nor pathologized is a critical aspect of developing a healthy sense of self in emerging adulthood. More than merely challenging the status quo, these relationships with adults in positions of power (relative to the queer young person) may also be seen as instrumental in the young person stabilising emotionally and working toward the fulfilment of their personal and career objectives. The implications for researchers can only be hypothesised within the limitations of the study. This was an extremely difficult group to recruit to participate in the study. A case study approach was therefore utilised to make meaning and visibilize the stories of Liam and Tim which provided an in-depth understanding of the lived experience of being in out-of-home care for queer young people. A clear implication for future research involving this cohort would be to strategize the recruitment strategy. A more formalised research partnership with an LGBTQ + support agency may be of value.

\section{Limitations of the Study}

Findings from this case study do not suggest that they are generalizable; however, they do suggest a need for a larger-scale study and the untapped stories within this community of young people in out-of-home care.

\section{Conclusion}

This study explored the lived experience of two queer young adults who had each spent part of their lives growing up in out-of-home care in Australia. Theories of minority stress and social capital were employed to provide a critical analysis of the challenges faced and, conversely, the impact of linking social capital that may facilitate the development of helpful resources and connections. The findings suggest that sustained experiences of minority stress can be overwhelming, rendering young queer people unable to explore their developing sexual and/or gender identity in the context of simply "trying to survive" in out-of-home care. The findings suggest also suggest that the presence, of trustworthy adults who challenge dominant heteronormative assumptions and work to support queer young people can be experienced as stabilising and restorative.

Author Contribution LMc- funding acquisition, conceptualization, methodology, data collection, formal analysis, supervision, writing original draft. KG- methodology, data collection, formal analysis, writing original draft, review and editing. NC- data collection, formal analysis, review and editing. JM- funding acquisition, conceptualization, review and editing.

Funding This project was funded by the NSW Department of Communities and Justice. Declaration: On behalf of all authors, the corresponding author states that there is no conflict of interest.

\section{Declarations}

Conflict of interest None.

\section{References}

Australian Institute of Family Studies. (2019). LGBTIQA+ Glossary of terms. Retrieved from https://aifs.gov.au/cfca/publications/lgbtiqcommunities

Barker, J., \& Thomson, L. (2015). Helpful Relationships with Service Users: Linking Social Capital. Australian Social Work, 68(1), 130-145. https://doi.org/10.1080/0312407X.2014.905795 
Bayliss, T. A. (2014). The art of seeing Aboriginal Australia's queer potential. The Conversation. Retrieved from https:// theconversation.com/the-art-of-seeing-aboriginal-australiasqueer-potential-25588

Beek, M., \& Schofield, G. (2004). Providing a secure base: Tuning in to children with severe learning difficulties in long-term foster care. Adoption \& Fostering, 28(2), 8-19. Retrieved from https://doi.org/ $10.1177 / 030857590402800203$

Bowleg, L., Huang, J., Brooks, K., Black, A., \& Burkholder, G. (2003). Triple jeopardy and beyond: Multiple minority stress and resilience among Black lesbians. Journal of Lesbian Studies: Trauma, Stress, and Resilience Among Sexual Minority Women: Rising Like the Phoenix, 7(4), 87-108. Retrieved from https://doi.org/ 10.1300/J155v07n04_06

Brandon-Friedman, R. A., Pierce, B., Wahler, E., Thigpen, J., \& Fortenberry, J. D. (2020). Sexual identity development and sexual well-being: Differences between sexual minority and non-sexual minority former foster youth. Children and Youth Services Review, 117.

Braun, V., \& Clarke, V. (2006). Using thematic analysis in psychology. Qualitative Research in Psychology, 3(2), 77-101. https://doi.org/10. 1191/1478088706qp063oa

Cameron, N., McPherson, L., Gatwiri, K., \& Parmenter, N. (2019). The Needs of LGBTIQ Young People in Out of Home Care. Research Brief. Centre for Excellence in Therapeutic Care, Australia.

Chevrette, R. (2013). Outing heteronormativity in interpersonal and family communication: Feminist applications of queer theory "Beyond the Sexy Streets". Communication Theory, 23(2), 170190. Retrieved from https://doi.org/10.1111/comt.12009

Cochran, B. N., Stewart, A. J., Ginzler, J. A., \& Cauce, A. M. (2002). Challenges faced by homeless sexual minorities: Comparison of gay, lesbian, bisexual, and transgender homeless adolescents with their heterosexual counterparts. American Journal of Public Health, 92(5), 773-777.

Cook, C., \& Cohen, C. (2018). 20 years of advocating for LGBTQ youth in out-of-home care. American Bar Association. Retrieved from https:/www.americanbar.org/groups/litigation/ committees/childrens-rights/articles/2018/spring2018-20-yearsadvocating-lgbtq-youth-out-of-home-care/

Coolhart, D., \& Brown, M. T. (2017). The need for safe spaces: Exploring the experiences of homeless LGBTQ youth in shelters. Children and Youth Services Review, 82, 230-238. Retrieved from https://doi.org/10. 1016/j.childyouth.2017.09.021

CREATE (2021) CREATEing Equality. Working with Children and Young People in Care who Idenitify as LGBTIQ. Downloaded on 14 May 2021 @ https://create.org.au/lgbt-resources/

Gatwiri, K., Mcpherson, L., Mcnamara, N., Mitchell, J., \& Tucci, J. (2018). From adversity to stability to integration: How one Australian program is making a difference in therapeutic foster care. Journal of Child Adolescent Trauma, 12(3), 1-12.

Jenkins, D., \& Johnston, L. B. (2004). Unethical treatment of gay and lesbian people with conversion therapy. Families in Society, 85(4), 557-561. Retrieved from https://doi.org/10.1606/1044-3894.1846

Kelleher, C. (2009). Minority stress and health: Implications for lesbian, gay, bisexual, transgender, and questioning (LGBTQ) young people. Counselling Psychology Quarterly, 22(4), 373-379. Retrieved from https://doi.org/10.1080/09515070903334995

Mallon, G. P. (1992). Gay and no place to go: Assessing the needs of gay and lesbian adolescents in out-of-home care settings. Child welfare, 71(6), 547-556. Retrieved from https://ezproxy.scu.edu. $\mathrm{au} /$ login?url=https://search.ebscohost.com/login.aspx?direct $=$ true $\& \mathrm{db}=$ mnh $\& A N=1424947 \&$ site $=$ ehost-live

Mallon, G. P., Aledort, N., \& Ferrera, M. (2002). There's no place like home: Achieving safety, permanency, and well-being for lesbian and gay adolescents in out-of-home care settings. Child welfare, 81(2), 407-439. Retrieved from https://ezproxy.scu.edu.au/login? url=https://search.ebscohost.com/login.aspx?direct $=$ true $\& \mathrm{db}=$ mnh\&AN $=12014475 \&$ site $=$ ehost-live

Mallon, G. P., \& Woronoff, R. (2006). Busting out of the child welfare closet: Lesbian, gay, bisexual, and transgenderaffirming approaches to child welfare. Child Welfare, 85(2), $115-122$.

McCormick, A., Schmidt, K., \& Terrazas, S. (2017). LGBTQ youth in the child welfare system: An overview of research, practice, and policy. Journal of Public Child Welfare, 11(1), 27-39. https://doi. org/10.1080/15548732.2016.1221368

McNair, R., Andrews, C., Parkinson, S., \& Dempsey, D. (2017). LGBTQ homelessness: Risks, resilience, and access to services in Victoria. Retrieved from http://www.lgbtihomeless.org.au/wpcontent/uploads/2018/04/LGBTQ-Homelessness-project-Finalreport-September-2017-Final_.pdf

McPherson, L., Gatwiri, K., Tucci, J., Mitchell, J., \& Macnamara, N. (2018). A paradigm shift in responding to children who have experienced trauma: The Australian treatment and care for kids program. Children and Youth Services Review, 94, 525-534. Retrieved from https://doi.org/10.1016/j.childyouth.2018.08.031

Mendes P., \& Snow, P. (2016). Young People Transitioning from Out of Home Care Palgrave Macmillan 978-1-137-55638-7

Meyer, I. H. (2003). Prejudice, social stress, and mental health in lesbian, gay, and bisexual populations: Conceptual issues and research evidence. Psychological Bulletin, 129(5), 674.

Oakley, S., \& Bletsas, A. (2018). The experiences of being a young LGBTIQ and homeless in Australia: Re-thinking policy and practice. Journal of Sociology, 54(3), 381-395.

Ragg, D. M., Patrick, D., \& Ziefert, M. (2006). Slamming the closet door: Working with gay and lesbian youth in care. Child welfare, 85(2), 243-265. Retrieved from https://ezproxy.scu.edu.au/login? url=https://search.ebscohost.com/login.aspx?direct=true $\& \mathrm{db}=$ mnh\&AN $=16846114 \&$ site $=$ ehost-live

Ryan, C., Huebner, D., Diaz, R. M., \& Sanchez, J. (2009). Family rejection as a predictor of negative health outcomes in white and latino lesbian, gay, and bisexual young adults. Pediatrics, 123(1), 346-335. Retrieved from https://doi.org/10.1542/peds.2007-3524

Schofield, G., Cossar, J., Ward, E., Larsson, B., \& Belderson, P. (2019). Providing a secure base for LGBTQ young people in foster care: The role of foster carers. Child \& Family Social Work, 24(3), 372-381.

Stanton-Salazar, R. D. (2011). A Social Capital Framework for the Study of Institutional Agents and Their Role in the Empowerment of Low-Status Students and Youth Youth and Society, 43(3), 10661109. https://doi.org/10.1177/0044118X10382877

The Center for the Study of Social Policy. (2016). Out of the shadows: Supporting LGBTQ youth in child welfare through cross-system collaboration. Retrieved from https://cssp.org/wp-content/uploads/ 2018/08/Out-of-the-Shadows-Supporting-LGBTQ-youth-in-childwelfare-through-cross-system-collaboration-web.pdf

Wilson, B. D., Cooper, K., Kastanis, A., \& Nezhad, S. (2014). Sexual and gender minority youth in foster care: Assessing disproportionality and disparities in Los Angeles.

Woronoff, R., Estrada, R., Sommer, S., Marzullo, M., Ryan, C., Downs, A. C., Ambroz, H. J. D., \& Karys, P. (2006). Out of the margings: A report on regional listening forums highlighting the experiences of lesbian, gay, bisexual, transgender, and questioning youth in care. Retrieved from https://www.lambdalegal.org/sites/default/ files/publications/downloads/out-of-the-margins.pdf

Yin, R. K. (2009). Case study research: Design and methods (4th ed.). Sage.

Publisher's Note Springer Nature remains neutral with regard to jurisdictional claims in published maps and institutional affiliations. 